\title{
EDITORIAL
}

\section{COPD: CardiOPulmonary Disease?}

\author{
Steven M. Kawut
}

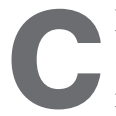
hronic obstructive pulmonary disease (COPD) is defined by the presence of airflow limitation which is not fully reversible, but COPD encompasses numerous phenotypes [1]. Phenotypes are the products of genetic and environmental interactions, with "environment" being broadly defined. The systematic measurement and analysis of clinical and other qualitative and quantitative traits may refine COPD phenotypes, features of which may be shared between different disease states [2, 3]. HAN et al. [4] have defined clinical phenotypes in COPD as "a single or combination of disease attributes that describe differences between individuals with COPD as they relate to clinically meaningful outcomes (symptoms, exacerbations, response to therapy, rate of disease progression, or death)." Identification of such phenotypes would not only facilitate outcome prediction and "personalised" treatment, but also improve the understanding of critical biological and mechanistic disease pathways. The systemic impact of COPD has led to consideration of extrapulmonary disease manifestations in recent efforts to construct these phenotypes.

This issue of the European Respiratory Journal contains one of two recent studies that focus on cardiovascular phenotyping in COPD [5, 6]. HuRDMAN et al. [5] have carefully evaluated the phenotype of severe pulmonary hypertension $(\mathrm{PH})$ (mean pulmonary artery pressure $\geqslant 40 \mathrm{mmHg}$ ) in COPD (PH-COPD) in comparison to the mild-moderate $\mathrm{PH}$ phenotype in a prospective cohort of patients referred to a specialty centre over almost a decade [7]. Echocardiography, spirometry and lung computed tomography (CT) imaging were performed with standardised interpretation. 59 patients with severe $\mathrm{PH}$ COPD were compared to 42 patients with mild-moderate $\mathrm{PH}$ COPD, with complete follow-up in all. Patients with severe PH-COPD had worse oxygenation and a lower diffusing capacity for carbon monoxide (DLCO), but higher forced expiratory volume in $1 \mathrm{~s}$ (FEV1), forced vital capacity (FVC), and FEV1/FVC ratio compared to those with mild-moderate PH-COPD. Patients with severe PH-COPD had higher right atrial pressure, lower cardiac index, higher pulmonary vascular resistance, and more limited exercise capacity. Interestingly, there were no differences in CT measures of emphysema or fibrosis between the groups. Most of those with

Pulmonary, Allergy and Critical Care Division, Penn Cardiovascular Institute, Center for Clinical Epidemiology and Biostatistics, Perelman School of Medicine at the University of Pennsylvania, Philadelphia, PA, USA

CORRESPONDENCE: S.M. Kawut, Pulmonary, Allergy and Critical Care Division, Perelman School of Medicine at the University of Pennsylvania, 423 Guardian Dr., Philadelphia, PA, USA. E-mail: kawut@upenn.edu severe $\mathrm{PH}-\mathrm{COPD}$ received targeted therapy for pulmonary arterial hypertension (PAH), but this group still had an increased risk of death. These data suggest that significant $\mathrm{PH}$ in the setting of COPD is a distinct phenotype, evidenced by more mild airflow obstruction (albeit with worse gas exchange) and worse outcomes. While it is unlikely that pulmonary artery pressure or other parameters are sufficiently discriminating to make definitive outcome predictions at the bedside, patients with severe PH-COPD (accompanied by lower cardiac index and DLCO) clearly do have a shorter survival.

Pigeonholing this syndrome is a challenge. Is the pronounced pulmonary vascular dysfunction in a subset of patients an extreme on the spectrum of the usual vascular response to local hypoxia, parenchymal destruction, and inflammation in COPD? Is the severe PH-COPD phenotype attributable to concomitant cardiopulmonary disease, such as sleep-disordered breathing? Or, is this phenotype more closely related to $\mathrm{PAH}$, which is traditionally diagnosed only in the absence of significant parenchymal lung disease? Could emphysema, its sequelae, or smoking actually trigger $\mathrm{PAH}$ ?

Some of these possibilities do not fit neatly into the World Health Organization (WHO) clinical categories of $\mathrm{PH}$, which guide scientific investigations, patient management and clinical trials, and have led to the approval of effective therapies (at least for some of the WHO PH categories) [8]. Patients with severe PH-COPD had greatly elevated pulmonary vascular resistance but a cardiac index that was relatively preserved compared to that of $\mathrm{PAH}$ patients at diagnosis. Studies have shown a circulatory limitation to exercise in severe PH-COPD, which is distinct from the ventilatory limitation seen in patients with mild-moderate PH-COPD [9]. Interrogation of these phenotypic features that cross the "usual" categorisations of PH may be informative in terms of mechanism and clinical approach, potentially grouping together types of $\mathrm{PH}$ which are currently neither considered of similar origin nor are thought to share common manifestations, treatments and outcomes. In this way, severe PH-COPD may indeed be more closely "related" to PAH than to COPD without $\mathrm{PH}$ or mild-moderate PH-COPD.

Prior cohort studies of patients with COPD suggest that about $1 \%$ of COPD patients have severe PH-COPD [10], which affected only 59 patients being referred to this centre over almost a decade (compared to 600 patients with PAH seen during the same period). That only 42 patients with mildmoderate PH-COPD (which is relatively common) were seen during the same period suggests a role for selective referral. It is possible that primary physicians were less likely to refer 
patients with severe $\mathrm{PH}$ in the setting of COPD (or mildmoderate $\mathrm{PH}-\mathrm{COPD}$ ) than to refer those with apparent $\mathrm{PAH}$, because of the assumption of limited therapeutic options for the group with COPD. The authors are careful to not draw inferences about incidence or prevalence of severe PH-COPD from their data; however, this appears to be a very rare phenotype, posing challenges to performing clinical trials solely in this population.

Importantly, the vast majority of patients with $\mathrm{PH}$ in COPD do not manifest this phenotype and do not respond to $\mathrm{PH}$ therapies. Previous trials of PAH medications in COPD, with or without $\mathrm{PH}$, have not only shown ineffectiveness, but have also suggested potential harm in some cases [11-14]. For most patients with PH-COPD, treatment with PAH drugs is simply not indicated. Whether these therapies may be effective in the very small subset of patients with severe PH-COPD is unknown, but they have been used in clinical practice.

The study by FreIXA et al. [6] focuses on right and left heart abnormalities visualised on transthoracic echocardiography in patients previously hospitalised for their first COPD exacerbation. Ischaemic cardiovascular disease and heart failure are important causes of death in patients with COPD, and acute ischaemic events are frequent in the setting of COPD exacerbations [15]. Cardiac dysfunction in COPD is often unrecognised and may be attributable to smoking and mechanistic pathways which are common to heart and lung diseases, including inflammation [16].

This research has several strengths, including a prospective design with performance of standardised assessments and centralised interpretation of echocardiography, what appears to be a generalisable study sample (at least to the local source population), and attention to quality assurance and quality control [17]. The investigators found a relatively high prevalence of left ventricular abnormalities, most commonly diastolic dysfunction. Right ventricular dilation was frequent, as was right ventricular diastolic dysfunction. Most left ventricular abnormalities were not associated with the severity of COPD, quality of life, or 6-min walking distance. Cluster analysis in this cohort suggested that the presence of cardiovascular disease (physician-diagnosed myocardial infarction or congestive heart failure) distinguished a phenotype of COPD characterised by higher fat-free mass, more inflammation, less lung dysfunction, higher lung density and more airways disease, and with a greater risk of cardiovascular and COPD admissions [18]. Another study of COPD phenotypes identified a subset of patients with more ischaemic heart disease and diabetes, more moderate airflow limitation, more bronchial thickening, and less alveolar destruction, but increased mortality [19]. Other investigators have shown similarly distinct "cardiac" COPD phenotypes associated with worse outcomes [20, 21].

The impact of pulmonary pathophysiology on cardiac structure and function is not surprising, considering the intertwining of the heart and lungs, and their juxtaposition in "close quarters" with continuously changing intravascular and intrathoracic pressures. The cardiovascular consequences of COPD may have a variety of mechanisms, including increased intrathoracic pressure, inflammation, hypoxaemia, or increased right ventricular and left ventricular afterload, and may interact with other environmental factors, such as smoking status $[22,23]$. Alternatively, the cardiovascular role in determining the risk of seemingly "lung-specific" outcomes in COPD (e.g. exacerbations) cannot be underestimated [24, 25].

This study suggests a common prevalence of biventricular abnormalities in COPD and highlights the possible usefulness of identifying a distinct COPD phenotype; however, there are some caveats. There were substantial missing data for many of the echocardiographic variables, which is not surprising considering the challenges that lung disease poses for adequate image windows. Almost $20 \%$ of the cohort was missing left atrial diameters, one-third was missing right ventricular diameters, and only approximately half had detected tricuspid regurgitation. Importantly, it is possible that the missingness may have depended on body habitus or severity of lung disease, making for possible differential information bias. These results demonstrate the difficulty of using transthoracic echocardiography in patients with COPD, even in this carefully designed and standardised protocol. Alternative cardiac measures, such as magnetic resonance imaging and CT, overcome these challenges, but pose other barriers in large multicentre studies.

Patients with cardiac disease (even if unbeknownst to the admitting physicians) may have been more likely to be hospitalised for their COPD exacerbation (rather than just receiving outpatient treatment), compared to patients without cardiac manifestations. Cardiac dysfunction might have even contributed to meeting the definition of an exacerbation. Therefore, inclusion in the cohort itself may have been predetermined by the existence of cardiac abnormalities, leading to selection bias and possible overestimates of the burden of cardiac dysfunction in the larger population. In addition, abnormal findings on echocardiography may have unclear clinical and therapeutic relevance. Therefore, the routine use of echocardiography in patients admitted with a COPD exacerbation does not have a known definitive benefit, making this strategy ideal for investigation in randomised clinical trials focused on clinical outcomes.

These two studies have identified potentially important cardiovascular contributions to COPD morbidity and mortality. Recent research has shown that heart failure, coronary artery disease and $\mathrm{PH}$ play prominent roles in the COPD "comorbidome", and may lead to an increased risk of death [26]. Future observational and interventional studies should incorporate measures of these important cardiovascular phenotypes, target patients with "high-risk" phenotypes, and test novel therapeutics which treat cardiovascular disease to impact on outcomes in COPD.

\section{STATEMENT OF INTEREST}

Conflict of interest information can be found alongside the online version of this article at www.erj.ersjournals.com

\section{REFERENCES}

1 Freimer N, Sabatti C. The human phenome project. Nat Genet 2003; 34: 15-21.

2 Hegele RA, Oshima J. Phenomics and lamins: from disease to therapy. Exp Cell Res 2007; 313: 2134-2143. 
3 Lanktree MB, Hassell RG, Lahiry P, et al. Phenomics: expanding the role of clinical evaluation in genomic studies. J Investig Med 2010; 58: 700-706.

4 Han MK, Agusti A, Calverley PM, et al. Chronic obstructive pulmonary disease phenotypes: the future of COPD. Am J Respir Crit Care Med 2010; 182: 598-604.

5 Hurdman J, Condliffe R, Elliot CA, et al. Pulmonary hypertension in COPD: results from the ASPIRE registry. Eur Respir J 2013; 41: 1292-1301.

6 Freixa X, Portillo K, Paré C, et al. Echocardiographic abnormalities in patients with COPD at their first hospital admission. Eur Respir J 2013; 41: 784-791.

7 Hurdman J, Condliffe R, Elliot CA, et al. ASPIRE registry: Assessing the Spectrum of Pulmonary hypertension Identified at a REferral centre. Eur Respir J 2012; 39: 945-955.

8 Simonneau G, Robbins IM, Beghetti M, et al. Updated clinical classification of pulmonary hypertension. J Am Coll Cardiol 2009; 54: Suppl. 1, S43-S54.

9 Boerrigter BG, Bogaard HJ, Trip P, et al. Ventilatory and cardiocirculatory exercise profiles in COPD: the role of pulmonary hypertension. Chest 2012; 142: 1166-1174.

10 Chaouat A, Bugnet AS, Kadaoui N, et al. Severe pulmonary hypertension and chronic obstructive pulmonary disease. Am J Respir Crit Care Med 2005; 172: 189-194.

11 Boeck L, Tamm M, Grendelmeier P, et al. Acute effects of aerosolized iloprost in COPD related pulmonary hypertension: a randomized controlled crossover trial. PloS One 2012; 7: e52248.

12 Lederer DJ, Bartels MN, Schluger NW, et al. Sildenafil for chronic obstructive pulmonary disease: a randomized crossover trial. COPD 2012; 9: 268-275.

13 Rietema H, Holverda S, Bogaard HJ, et al. Sildenafil treatment in COPD does not affect stroke volume or exercise capacity. Eur Respir J 2008; 31: 759-764.

14 Stolz D, Rasch H, Linka A, et al. A randomised, controlled trial of bosentan in severe COPD. Eur Respir J 2008; 32: 619-628.
15 McAllister DA, Maclay JD, Mills NL, et al. Diagnosis of myocardial infarction following hospitalisation for exacerbation of COPD. Eur Respir J 2012; 39: 1097-1103.

16 Macchia A, Rodriguez Moncalvo JJ, Kleinert M, et al. Unrecognised ventricular dysfunction in COPD. Eur Respir J 2012; 39: 51-58.

17 Balcells E, Antó JM, Gea J, et al. Characteristics of patients admitted for the first time for COPD exacerbation. Respir Med 2009; 103: 1293-1302.

18 Garcia-Aymerich J, Gómez FP, Benet M, et al. Identification and prospective validation of clinically relevant chronic obstructive pulmonary disease (COPD) subtypes. Thorax 2011; 66: 430-437.

19 Burgel PR, Paillasseur JL, Peene B, et al. Two distinct chronic obstructive pulmonary disease (COPD) phenotypes are associated with high risk of mortality. PloS One 2012; 7: e51048.

20 Burgel PR, Paillasseur JL, Caillaud D, et al. Clinical COPD phenotypes: a novel approach using principal component and cluster analyses. Eur Respir J 2010; 36: 531-539.

21 Burgel PR, Roche N, Paillasseur JL, et al. Clinical COPD phenotypes identified by cluster analysis: validation with mortality. Eur Respir J 2012; 40: 495-496.

22 Barr RG, Bluemke DA, Ahmed FS, et al. Percent emphysema, airflow obstruction, and impaired left ventricular filling. $N$ Engl J Med 2010; 362: 217-227.

23 Grau M, Barr R, Lima JA, et al. Percent emphysema and right ventricular structure and function: the MESA Lung and MESA-RV Studies. Chest 2013 [In press DOI: 10.1378/chest.12-1779].

24 Kessler R, Faller M, Fourgaut G, et al. Predictive factors of hospitalization for acute exacerbation in a series of 64 patients with chronic obstructive pulmonary disease. Am J Respir Crit Care Med 1999; 159: 158-164.

25 Wells JM, Washko GR, Han MK, et al. Pulmonary arterial enlargement and acute exacerbations of COPD. $N$ Engl J Med 2012; 367: 913-921.

26 Divo M, Cote C, de Torres JP, et al. Comorbidities and risk of mortality in patients with chronic obstructive pulmonary disease. Am J Respir Crit Care Med 2012; 186: 155-161. 